\title{
The integration of next-generation sequencing panels in the clinical cancer genetics practice: an institutional experience
}

\author{
Caitlin B. Mauer, MS, CGC', Sara M. Pirzadeh-Miller, MS, $\mathrm{CGC}^{2}$, Linda D. Robinson, MS, $\mathrm{CGC}^{3}$ \\ and David M. Euhus, MD, FACS ${ }^{4}$
}

Purpose: The advent of next-generation sequencing for cancer susceptibility genes holds promise for clinical genetics application, but the practical issues surrounding integration of this testing into the clinical setting have not been well addressed. This article describes the clinical experience of genetic counselors in an academic and community setting with next-generation sequencing cancer panels.

Methods: Between April 2012 and January 2013, 60 next-generation sequencing panels were ordered. A retrospective review was conducted to determine the indication for ordering the results of the tests and the patient management based on the results.

Results: Ten tests were canceled due to out-of-pocket costs or previously identified mutations. Among the 50 tests, 5 (10\%) showed a positive result. Moreover, 15 of the $50(30 \%)$ panels detected variant(s) of uncertain significance or variant(s) suspected benign.
Conclusion: We propose clinical guidelines for identifying highrisk patients who should be offered this testing. Our data support the National Comprehensive Cancer Network recommendations that next-generation sequencing be ordered as a second-tier test for high-risk individuals with cancer by trained cancer genetics providers. Literature review and expert knowledge should be used to create management plans for the identification of both positive and variants of uncertain significance results. Providers should be aware of limitations regarding reimbursement for testing and recommended management strategies.

Genet Med advance online publication 10 October 2013

Key Words: $B R C A$; genetic testing; genomics; next-generation sequencing; panel testing

\section{INTRODUCTION}

Hereditary cancer syndromes account for $\sim 5-10 \%$ of all cancer diagnoses. Genetic testing provides a powerful tool for stratifying individuals' cancer risks. Historically, a personal and/or family history of cancer combined with phenotypic clues has been used to indicate the genes that are most likely to have an identifiable mutation. The most likely genes were evaluated first with single tests followed by additional single gene tests if no mutation was identified and additional genes were part of the differential. With the recent advent of massive parallel sequencing, or next-generation sequencing (NGS), multiple genes can now be evaluated simultaneously.

Germline NGS panels are available for clinical diagnosis in several genetic subspecialties. Cardiomyopathy panels were one of the first NGS panels to be developed, and the cost effectiveness of sequencing many known and novel cardiomyopathy genes simultaneously for overlapping phenotypes of hypertrophic and dilated cardiomyopathies was quickly established. In a study evaluating 43 novel and known cardiomyopathy genes, deleterious mutations were identified in 6 out of 10 dilated and hypertrophic cardiomyopathy patients. ${ }^{1}$ However, numerous variants were also identified, creating difficulties for interpretation. More recently, NGS panels have been developed for other specialties including X-linked intellectual disability, neuromuscular disorders, and syndromic and nonsyndromic hearing loss. ${ }^{2}$

Several laboratories have recently released commercial NGS panels for hereditary cancer syndromes. Although highly penetrant genes (18- to 20 -fold cancer risk) are included on many of these panels, more commonly, the NGS panels include moderately penetrant genes (two- to fivefold cancer risk), with different laboratories offering a slightly varied menu of genes.

Detecting deleterious mutations or variants in these moderately penetrant genes can be challenging for clinicians, as currently there are no established clinical management guidelines. In addition, there are no clear guidelines for the identification of eligible patients for offering NGS panel testing, there are unresolved reimbursement issues, and management recommendations are lacking for moderate penetrance genes. In this article, we systematically assess our initial experience with NGS cancer panels ordered through Ambry Genetics with the intent of establishing institutional guidelines for ordering and interpreting these tests.

\section{MATERIALS AND METHODS}

A retrospective review was performed of all NGS cancer panels ordered by a large academic cancer genetics program between April 2012 and January 2013. Our program, which

\footnotetext{
${ }^{1}$ Department of Cancer Genetics, University of Texas Southwestern Medical Center, Dallas, Texas, USA; ${ }^{2}$ Department of Cancer Genetics, Moncrief Cancer Institute, University of Texas Southwestern Medical Center, Fort Worth, Texas, USA; ${ }^{3}$ Department of Cancer Genetics, University of Texas Southwestern Medical Center, Dallas, Texas, USA; ${ }^{4}$ Department of Surgery, Division of Surgical Oncology, University of Texas Southwestern Medical Center, Dallas, Texas, USA. Correspondence: Caitlin B. Mauer (caitlin.mauer@utsouthwestern.edu) 
evaluates genetic predisposition to cancers of all types, includes seven board-certified genetic counselors, divided between an academic center in Dallas, TX (UT Southwestern Simmons Comprehensive Cancer Center) and a community setting in Fort Worth, TX (Moncrief Cancer Institute). All NGS panels were ordered through Ambry Genetics including BreastNext (14 hereditary breast cancer genes), OvaNext (19 hereditary ovarian cancer genes), ColoNext (14 hereditary colon cancer genes), and CancerNext (22 hereditary cancer genes) (Table 1). ${ }^{3}$

Our initial criteria for ordering a panel were based loosely on recommendations from Ambry's white paper ${ }^{4}$ (disregarding panel testing on patients with breast cancer $<50$ alone), current National Comprehensive Cancer Network (NCCN) guidelines for the more common hereditary cancer syndromes, ${ }^{5}$ and our own professional experience with general indications for "highrisk" patients after negative first-tier genetic testing. Of note, at the time we offered this testing, genetic testing patents protected $B R C A 1$ and $B R C A 2$ from being tested on these panels.

A retrospective data review focused on understanding the specific indications for each test ordered and summarizing the results of the tests. As some patients' tests yielded multiple sequence changes, each test was categorized based on its most severe result (in order of severity: positive, variants of uncertain significance (VUS), variants suspected benign (VSB), and none

\begin{tabular}{|c|c|c|c|c|}
\hline Gene & BreastNext & OvaNext & ColoNext & CancerNext \\
\hline ATM & - & - & & - \\
\hline BARD1 & - & - & & - \\
\hline BRIP1 & • & • & & • \\
\hline MRE11A & - & - & & - \\
\hline NBN & • & • & & • \\
\hline RAD50 & - & - & & - \\
\hline RAD51C & • & • & & • \\
\hline PALB2 & • & • & & - \\
\hline STK11 & • & • & - & • \\
\hline CHEK2 & - & - & - & - \\
\hline PTEN & • & • & • & • \\
\hline TP53 & • & • & • & • \\
\hline $\mathrm{CDH} 1$ & • & • & • & • \\
\hline MUTYH & • & • & • & • \\
\hline MLH1 & & • & • & • \\
\hline MSH2 & & • & • & • \\
\hline MSH6 & & • & • & • \\
\hline EPCAM & & • & • & • \\
\hline PMS2 & & • & • & • \\
\hline$A P C$ & & & • & • \\
\hline BMPR1A & & & • & • \\
\hline SMAD4 & & & - & - \\
\hline
\end{tabular}

NGS, next-generation sequencing. $\bullet$ Indicates which NGS panels the corresponding genes are on. detected). We then identified the key factors involved in interpreting and acting on the test results, recognized reimbursement issues, and analyzed the overall utility of NGS testing within our clinical practice. From this retrospective data review and the expert opinion of our staff, we developed our own internal criteria for ordering and interpreting hereditary cancer NGS panels.

\section{RESULTS}

Among the 1,521 new cancer genetics patients evaluated during the study period, $1,233(81.1 \%)$ underwent genetic testing. NGS panels were ordered on 60 (4.9\%) of these patients. Fiftyfour of these $60(90 \%)$ NGS panels were ordered as a secondtier test after negative single-gene testing was performed for patients who met the NCCN criteria for genetic testing of any known hereditary cancer predisposition syndromes. The most common test ordered was the BreastNext panel $(n=37)$, followed by CancerNext $(n=12)$. Ten of the $60(16.7 \%)$ panels initially ordered were subsequently canceled; eight of the ten (80\%) cancellations were due to either insurance denial of coverage or an out-of-pocket cost deemed too high by the patient. The turn around time for results ranged from 81 to 162 days (mean: 102 days).

\section{Determination of who received testing}

All 37 BreastNext panels were ordered on patients with a personal history of breast cancer. Thirty-six of these 37 (97.3\%) patients had negative BRCA testing before ordering the BreastNext panel. All 36 patients had full gene sequencing; 30 patients also had deletion/duplication analysis known as BRCA Analysis Rearrangement Testing. Posttest BRCAPRO (a risk calculation model for hereditary breast cancer) calculations for these patients ranged from $<1$ to $95 \%$ (mean: $6.6 \%$ ). Four of the eight (50\%) ColoNext panels were ordered on patients with a personal history of colon cancer. Three of the eight (37.5\%) had colon cancer-associated genetic testing performed before the ordering of the ColoNext panel (one APC sequencing and deletion/duplication, one APC and $M Y H$ sequencing and deletion/duplication, and one MLH1/MSH2/MSH6/PMS2/EPCAM sequencing and deletion/duplication). MMRPro (a risk calculation model for hereditary colon cancer) calculations ranged from $<1$ to $78 \%$ (mean: $2.4 \%$ ) for these patients. All three OvaNext tests were ordered on patients affected with ovarian cancer, whereas 10 of the 12 (83.3\%) CancerNext panels were ordered on patients with a personal history of cancer.

\section{Test results}

Among the 1,233 single-gene tests ordered at our center in the study time frame, $131(10.6 \%)$ returned a deleterious result compared with 5 of the $50(10 \%)$ completed NGS panel tests. (The centers' annual mutation rate in the period of 6 years ranges from 12 to $21 \%$; mean: $15.3 \%$ ).

Positive mutations were identified on three BreastNext tests (RAD51C, CHEK2, and ATM) and two ColoNext tests (CHEK2 and MUTYH) (Table 2). VUSs and VSBs were identified on 15 of the 50 (30\%) completed tests: $22.6 \%$ (7/31) on BreastNext 
Table 2 Identified DNA changes found by NGS panels

\begin{tabular}{|c|c|c|c|}
\hline Gene & Panel type & $\begin{array}{l}\text { Sequence/amino } \\
\text { acid change }\end{array}$ & Classification \\
\hline$A P C$ & CancerNext & c.3468_3470delAGA & VUS \\
\hline ATM & CancerNext & p.Y1442H & VUS \\
\hline ATM & BreastNext & p.K1992T & VUS \\
\hline ATM & BreastNext & p.Y1475C & VUS \\
\hline ATM & CancerNext & p.M1006V & VUS \\
\hline ATM & BreastNext & p.Y1124X & Positive \\
\hline ATM & CancerNext & p.L1420F & VSB \\
\hline BARD1 & BreastNext & p.Q11H & VSB \\
\hline BARD1 & BreastNext & p.C557s & VSB \\
\hline BARD1 & BreastNext & p.1738V & VSB \\
\hline BARD1 & BreastNext & p.1738V & VSB \\
\hline BRIP1 & CancerNext & p.V193I & VSB \\
\hline BRIP1 & BreastNext & p.D1138Y & VUS \\
\hline $\mathrm{CDH} 1$ & BreastNext & p.R492T & VUS \\
\hline CHEK2 & ColoNext & c.1100delC & Positive \\
\hline CHEK2 & BreastNext & c.1263delT & Positive \\
\hline CHEK2 & BreastNext & p.H143R & VUS \\
\hline MSH6 & OvaNext & p.S503C & VUS \\
\hline MUTYH & ColoNext & p.K449N & Positive \\
\hline MUTYH & ColoNext & c. $505-4 A>G$ & VSB \\
\hline PMS2 & CancerNext & p.I18V & VUS \\
\hline PMS2 & ColoNext & p.L166P & VUS \\
\hline PMS2 & CancerNext & p.D699H & VUS \\
\hline RAD51C & BreastNext & p.R193X & Positive \\
\hline RAD51C & BreastNext & р.T287A & VSB \\
\hline STK11 & OvaNext & p.S404F & VUS \\
\hline
\end{tabular}

NGS, next-generation sequencing; VSB, variant suspected benign; VUS, variant of uncertain significance.

panels, $20 \%(1 / 5)$ on ColoNext panels, $66.6 \%(2 / 3)$ on OvaNext panels, and $45.5 \%(5 / 11)$ on CancerNext panels (Figure 1).

\section{Management decisions}

Of the 50 completed tests, $30(60 \%)$ did not affect management decisions, 15 (30\%) introduced uncertainty regarding the patients' cancer risks, and 5 (10\%) directly influenced management decisions. The CHEK2 positive patients were counseled according to the management guideline previously established at our institution, whereas the RAD51C, ATM, and MUTYH mutation results prompted a thorough review of the literature to determine cancer risks and appropriate management recommendations.

\section{DISCUSSION}

\section{Patient selection}

Before the recent recommendations from the NCCN addressing NGS panel testing as a second-tier test ordered by cancer genetic professionals, our center established generalized

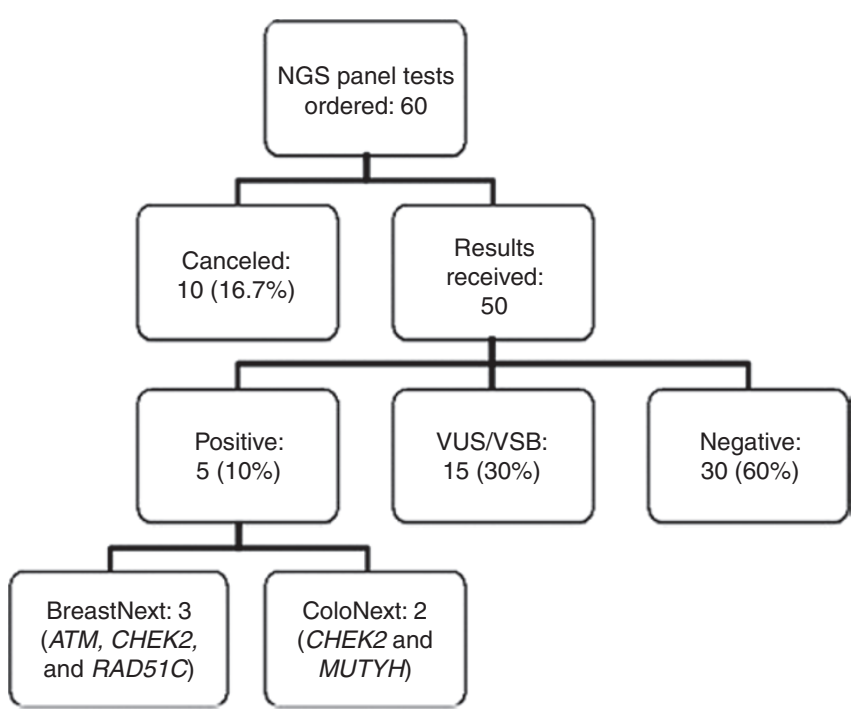

Figure 1 Flowchart of next-generation sequencing (NGS) testing and results. Ten tests were canceled. Positive mutations were identified on three BreastNext tests and two ColoNext tests. Variants of uncertain significance (VUS) and variants suspected benign (VSB) were identified on 15 of 50 (30\%) completed tests.

criteria to uniformly identify appropriate patients for NGS panel testing, which became our study cohort. Our data show a $10 \%$ mutation rate within this select group of patients. After retrospective review, we then devised more stringent criteria to aid our cancer genetics professionals in determining when NGS panel testing should be offered to patients (Table 3).

The retrospective application of our proposed clinical criteria to our study population indicates that we should not have offered testing to 11 patients. In fact, the more stringent criteria continue to capture our mutation-positive patients. Therefore, our proposed criteria would increase our overall detection rate on NGS panels (which excluded BRCA1 and BRCA2 at the time of testing) to $11.9 \%$, which is more comparable with mutation rates of $5,{ }^{6} 12,{ }^{7}$ and $24 \%{ }^{8}$ identified in NGS panel studies with highly selective patient populations that included BRCA testing. On the basis of these mutation rates, it is likely that NGS panel testing would yield a lower mutation rate if offered to an unselected genetics referral population.

\section{Interpretation}

Positives. In the context of our positive results, we determined that NGS panels successfully identified mutations in moderately penetrant genes that may not have otherwise been tested given the patient's clinical history. The lifetime cancer risks associated with mutations in many of these moderately penetrant hereditary cancer genes are still uncertain. Furthermore, the types of cancer associated with mutations and the burden of certain mutations in men need further research. ${ }^{9}$ This ambiguity adds to the difficulty of interpretation in the clinical setting when advising patients about management options. While the NCCN guidelines can be used to manage certain 


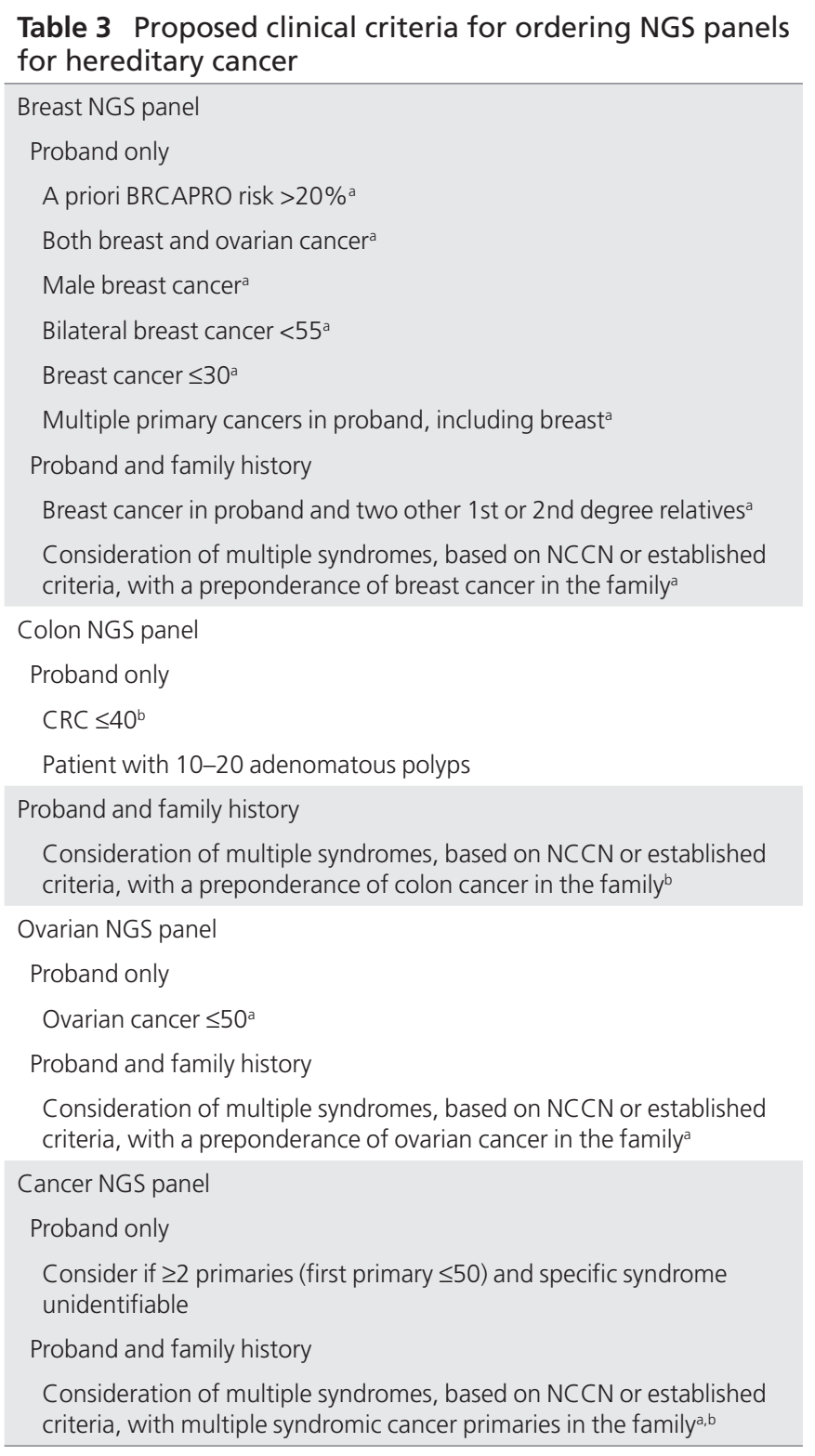

BART, BRCA analysis rearrangement testing; CRC, colorectal cancer; IHC, immunouhistochemical; NCCN, National Comprehensive Cancer Network; NGS, next-generation sequencing; MSI, microsatellite instability.

aAfter negative BRCA comprehensive sequencing and BART. 'b With normal tumor IHC/MSI or if tumor IHC/MSI is unavailable.

mutation carriers (e.g., Lynch syndrome patients, hereditary breast and ovarian cancer syndrome patients, etc.), there are no established guidelines for managing patients with mutations in newly identified genes. ${ }^{5}$

To manage our patients with positive NGS results, we turned to the literature and our knowledge of genetic mechanisms to help in determining the cancer risks associated with mutations in specific genes. In addition, a careful assessment of the threegeneration pedigree was often helpful for estimating familyspecific penetrance. Management recommendations were generally made based on other known syndromes with comparable risks.
Our approach to a BreastNext RAD51C mutation illustrates this point. A review of all available literature suggested that RAD51C mutations can confer up to a 33\% lifetime risk of breast cancer and a $>9 \%$ lifetime risk of ovarian cancer, ${ }^{9-11}$ although there is some evidence to suggest that cancer risks are similar to those associated with $B R C A 2$ mutations. Because the $R A D 51 C$ gene is integral to the BRCA2 pathway, we were able to modify our $B R C A$-positive recommendations for highrisk screening, chemoprevention, and/or risk-reducing surgeries and apply them to the patient and her family members. However, additional cancer risks and the effects of RAD51C mutations in males remain unknown, so management recommendations for family members were approached conservatively. ${ }^{9}$ This patient has opted for bilateral prophylactic mastectomy and risk-reducing oophorectomy.

A similar approach was taken to manage our ATM heterozygous patient. Literature cites a $28-50 \%$ lifetime risk for breast cancer in ATM heterozygote mutation carriers, with an additional increased risk if a first-degree relative is affected with breast cancer. ${ }^{12-14}$ Using high-risk breast cancer screening and management guidelines, we recommended breast magnetic resonance imaging and ultrasounds and discussed the availability of prophylactic surgical interventions. Although current literature is conflicting, given that ATM carriers may have an increased sensitivity to radiation, we recommended that mammography and X-rays should only be considered if no alternative method could be considered for a particular therapy, treatment, or management option. ${ }^{15,16}$ Evidence suggests that specific mutations within the ATM gene may increase the risks for other cancers; we recommended family history to be used to guide screening and management recommendations for these other cancers. ${ }^{17}$ Finally, given that homozygous ATM mutations can cause ataxia telangiectasia, preconception/prenatal genetic counseling was recommended for any ATM carrier of reproductive age.

Because genes such as ATM and MUTYH have additional implications if homozygous mutations are inherited, we felt it pertinent to disclose heterozygous mutations in $M U T Y H$, given the reproductive risks in addition to the postulated cancer risks. However, given that there are NCCN guidelines for managing these patients, details regarding our recommendations are not outlined in this article.

While our knowledge about the cancer risks associated with mutations in moderately penetrant genes continues to grow, genotype-specific literature can make interpretation difficult when a patient does not have a common mutation. Many published articles about CHEK2 are in regards to the common Eastern European 1100delC mutation. These data were used in conjunction with published data for high-risk women to create modified recommendations for our CHEK2 c.1263delT patient. These recommendations included annual magnetic resonance imaging in addition to mammography, given the up to $25 \%$ lifetime risk of breast cancer and the consideration of chemoprevention, because many CHEK2 breast cancers are estrogenreceptor positive. ${ }^{18,19}$ As prostate cancer risks are elevated in individuals with the 1100delC mutation, CHEK2-positive men 
with a first-degree relative with prostate cancer are recommended to begin prostate cancer screening 5-10 years before the earliest diagnosis in a close relative. ${ }^{20,21}$ Given that CHEK2 mutations have been seen to increase risks of thyroid, ovarian, kidney, and colon cancer, we advised screening for these cancers in the context of a positive family history of these cancers. $^{22-24}$ The patient elected care at an outside institution and her follow-up is unknown.

Variants. As Ambry has previously reported, we found that NGS cancer panels frequently detect VUSs. As more panel tests are ordered and the frequency of these detected variants increases, we expect the VUS rate to drop as the variants become reclassified. Until then, providers should disclose the possibility of detecting variants when counseling and consenting patients for panel testing and should maintain patient contact information so that patients can be notified when variants are reclassified. In addition, providers should be ready to dedicate time for interpreting VUSs in the context of the proband's personal and family history and help in enrolling patients in further VUS family studies to help in reclassifying variants. As NGS panels expand in availability, providers need to be prepared for an increase in case management time and the associated long-term follow-up of these VUSs in regards to reclassification.

The complexities of VUSs are exemplified in the case of a 64-year-old Caucasian woman presenting for colon cancer risk assessment. Her personal history consisted of invasive ductal carcinoma and ductal carcinoma in situ of the right breast at the age of 51 years. She reported a three-generation maternal family history of colon and uterine cancers, suspicious for Lynch syndrome. However, the family did not meet Amsterdam II Criteria due to a lack of a cancer diagnosis in $<50$ years of age (the youngest diagnosis was at the age of 51 years). The patient's CancerNext panel identified two PMS2 VUSs and an ATM suspected benign variant.

While these variants may play a role in the patient's family history, at this time there is not enough data about these variants to change any medical recommendations on these findings alone. To further understand the genetic risks, we obtained the proband's mother's colon tumor and performed immunouhistochemical staining. The staining showed normal expression of all mismatch repair proteins, greatly reducing the likelihood of Lynch syndrome. However, further germline testing of the proband's mother and other affected relatives (deceased) would have helped to determine whether these PMS2 variants are in cis or trans and if they may be linked to a history of colon or endometrial cancer. Lacking more specific information about these variants, recommendations of colonoscopies every 3-5 years were made for the proband based on her family history and the lack of evidence of Lynch syndrome. Eventually these variants will be reclassified, which may transform these results from being uninformative to becoming useful in the clinical setting. This case demonstrates the complexities of NGS panels and illustrates the NCCN recommendations that NGS panels only be ordered in consultation with cancer genetic professionals.

\section{Financial implications}

We found that third-party coverage of the testing played a significant role in the patient's desire to undergo NGS testing. Eight patients (13.3\%) decided to cancel their NGS panel testing after learning of their out-of-pocket costs. We expect that as NGS panel testing becomes more common in the clinical cancer genetics setting, insurance companies will create their own criteria regarding coverage of these tests. Until then, preauthorization of testing and discussions about financial costs to the patient are imperative.

We expect to encounter difficulties in obtaining insurance coverage for high-risk surveillance and/or risk-reducing surgeries for patients identified to have mutations in rare, likely moderate penetrance genes with little available information. Without standard guidelines informing medical management for these patients, health-care providers will need to work actively to help patients in obtaining financial coverage for the proper surveillance and/or surgical management of their cancer risks.

Currently there are no cost-effectiveness studies available for NGS panel genetic testing. With limited health-care dollars, there is a need to develop a strategy for efficient ordering of NGS panels to screen for hereditary cancer syndromes. While whole-exome/genome sequencing will soon allow for population screening for hereditary conditions, this is not economically feasible at this time. Recently, the Supreme Court ruled to overturn the legality of gene patents, and several laboratories have already started to market BRCA1 and BRCA2 testing as a stand-alone test and within the NGS panel setting. With this, we anticipate NGS panels will start to be marketed as first-tier tests. However, cancer genetic professionals must strongly consider the economic utility of NGS panels in low-risk patients compared with the overall costs of NGS testing. Difficulties in interpretation are clearly greater in patients whose pedigree analysis suggests low or moderate penetrance. We anticipate our proposed criteria will aid cancer genetic professionals in identifying patients for NGS panel testing, and we maintain that NGS panel testing should be directed toward patients who have previously tested negative for highly penetrant genetic mutations.

As NGS panel testing becomes more mainstream, we will rapidly gain a better understanding of the true cancer risks associated with mutations in genes rarely tested previously. For now, we recommend that NGS panel testing should be ordered only in a highly selective patient population to obtain actionable results. We concur with the NCCN recommendations to order NGS panels as a second-tier test, and we propose criteria to help in aiding cancer genetic professionals to identify these highrisk patients. Until we gain more knowledge, positive, negative, and variant results should be interpreted in the context of the patient's personal and family history. In addition, third-party payors will need to rapidly adjust their coverage guidelines in order for the patients to obtain genetic testing and the appropriate medical management based on their test results.

\section{DISCLOSURE}

The authors declare no conflicts of interest. 


\section{REFERENCES}

1. Meder B, Haas J, Keller A, et al. Targeted next-generation sequencing for the molecular genetic diagnostics of cardiomyopathies. Circ Cardiovasc Genet 2011:4:110-122.

2. Lin X, Tang W, Ahmad S, et al. Applications of targeted gene capture and nextgeneration sequencing technologies in studies of human deafness and other genetic disabilities. Hear Res 2012;288:67-76.

3. Ambry Genetics, 2013. Hereditary cancer panels. http://ambrygenetics.com/ next-gen-cancer-panels Accessed 1 July 2013.

4. Ambry Genetics, 2012. The case for clinical adoption of hereditary breast cancer panel testing. http://www.ambrygen.com/sites/default/files/pdfs/cancer\%20 forms/BreastNext_WhitePaper_100412.pdf Accessed 1 July 2013.

5. National Comprehensive Cancer Network, Inc., 2013. Genetic/Familial HighRisk Assessment: Breast and Ovarian, Version 2.2013. http://www.nccn.org/ professionals/physician_gls/pdf/genetics_screening.pdf Accessed 8 March 2013.

6. Pennington KP, Walsh $T$, Lee $M$, et al. BRCA1, TP53, and CHEK2 germline mutations in uterine serous carcinoma. Cancer 2013;119:332-338.

7. Walsh T, Casadei S, Coats KH, et al. Spectrum of mutations in BRCA1, BRCA2, CHEK2, and TP53 in families at high risk of breast cancer. JAMA 2006;295:1379-1388.

8. Walsh T, Casadei S, Lee MK, et al. Mutations in 12 genes for inherited ovarian, fallopian tube, and peritoneal carcinoma identified by massively parallel sequencing. Proc Natl Acad Sci USA 2011;108: 18032-18037.

9. Silvestri V, Rizzolo P, Falchetti M, et al. Mutation screening of RAD51C in male breast cancer patients. Breast Cancer Res 2011;13:404.

10. Loveday C, Turnbull C, Ruark E, et al.; Breast Cancer Susceptibility Collaboration (UK). Germline RAD51C mutations confer susceptibility to ovarian cancer. Nat Genet 2012;44:475-476; author reply 476.

11. Meindl A, Hellebrand $H$, Wiek $C$, et al. Germline mutations in breast and ovarian cancer pedigrees establish RAD51C as a human cancer susceptibility gene. Nat Genet 2010;42:410-414.

12. Ahmed M and Rahman N. ATM and breast cancer susceptibility. Hum Mutat 2006; 27:1122-1128.
13. Renwick A, Thompson D, Seal S, et al.; Breast Cancer Susceptibility Collaboration (UK). ATM mutations that cause ataxia-telangiectasia are breast cancer susceptibility alleles. Nat Genet 2006;38:873-875.

14. Bernstein JL, Teraoka S, Southey MC, et al. Population-based estimates of breast cancer risks associated with ATM gene variants C.7271T $>\mathrm{G}$ and c. 1066$6 \mathrm{~T}>\mathrm{G}$ (IVS10-6T>G) from the Breast Cancer Family Registry. Hum Mutat 2006;27:1122-1128.

15. Gutiérrez-Enríquez S, Fernet $M$, Dörk T, et al. Functional consequences of ATM sequence variants for chromosomal radiosensitivity. Genes Chromosomes Cancer 2004;40:109-119.

16. Mao JH, Wu D, DelRosario R, Castellanos A, Balmain A, Perez-Losada J. Atm heterozygosity does not increase tumor susceptibility to ionizing radiation alone or in a p53 heterozygous background. Oncogene 2008;27:6596-6600.

17. Angèle $S$, Falconer $A$, Edwards SM, et al. ATM polymorphisms as risk factors for prostate cancer development. Br J Cancer 2004;91:783-787.

18. CHEK2 Breast Cancer Case-Control Consortium. CHEK2*1100delC and susceptibility to breast cancer: a collaborative analysis involving 10,860 breast cancer cases and 9,065 controls from 10 studies. Am J Hum Genet 2004; 74:1175-1182.

19. Fisher B, Costantino JP, Wickerham DL, et al. Tamoxifen for the prevention of breast cancer: current status of the National Surgical Adjuvant Breast and Bowel Project P-1 study. J Natl Cancer Inst 2005;97:1652-1662.

20. Cybulski C, Wokolorczyk D, Huzarski T, et al. A large germline deletion in the Chek2 kinase gene is associated with an increased risk of prostate cancer. J Med Genet 2006;43:863-866.

21. Schröder FH, Hugosson J, Roobol MJ, et al.; ERSPC Investigators. Screening and prostate-cancer mortality in a randomized European study. N Eng/ J Med 2009;360:1320-1328.

22. Cybulski C, Górski B, Huzarski T, et al. CHEK2 is a multiorgan cancer susceptibility gene. Am J Hum Genet 2004;75:1131-1135.

23. Szymanska-Pasternak J, Szymanska A, Medrek K, et al. CHEK2 variants predispose to benign, borderline and low-grade invasive ovarian tumors. Gynecol Oncol 2006;102:429-431.

24. Xiang HP, Geng XP, Ge WW, Li H. Meta-analysis of CHEK2 1100delC variant and colorectal cancer susceptibility. Eur J Cancer 2011;47:2546-2551. 